An embedded mesh method for treating overlapping finite element meshes

J. D. Sanders, M. .A. Puso

March 15, 2011

International Journal for Numerical Methods in Engineering 
This document was prepared as an account of work sponsored by an agency of the United States government. Neither the United States government nor Lawrence Livermore National Security, LLC, nor any of their employees makes any warranty, expressed or implied, or assumes any legal liability or responsibility for the accuracy, completeness, or usefulness of any information, apparatus, product, or process disclosed, or represents that its use would not infringe privately owned rights. Reference herein to any specific commercial product, process, or service by trade name, trademark, manufacturer, or otherwise does not necessarily constitute or imply its endorsement, recommendation, or favoring by the United States government or Lawrence Livermore National Security, LLC. The views and opinions of authors expressed herein do not necessarily state or reflect those of the United States government or Lawrence Livermore National Security, LLC, and shall not be used for advertising or product endorsement purposes. 


\title{
An Embedded Mesh Method for Treating Overlapping Finite Element Meshes
}

\author{
Jessica Sanders ${ }^{1, *}$, Michael A. Puso ${ }^{1}$
}

\begin{abstract}
A new technique for treating the mechanical interactions of overlapping finite element meshes is presented. Over the years, many different names have been given to methods treating overlapping meshes, here we will refer to them all as embedded mesh methods. Such methods can be useful for numerous applications e.g., fluid-solid interaction with a superposed meshed solid on an Eulerian background fluid grid or solid-solid interaction with a superposed meshed particle on a matrix background mesh, etc. In this work, we consider the interaction of two elastic domains: one mesh is the foreground and defines the surface of interaction, the other is a background mesh and is often a structured grid. Many of the previously proposed methods employ surface defined Lagrange multipliers or penalties to enforce the boundary constraints. It has become apparent that these methods will cause mesh locking under certain conditions. Appropriately applied, the Nitsche method can overcome this locking $([1])$, but, in its canonical form, is generally not applicable to non-linear materials such as hyperelastics. The relationship between interior point penalty, discontinuous Galerkin and Nitsche's method is well known (see [2]). Based on this relationship, a nonlinear theory analogous to the Nitsche method is proposed to treat nonlinear materials in an embedded mesh. Here, a DG derivative based on a lifting of the interface surface integrals provides a consistent treatment for non-linear materials and demonstrates good behavior in example problems.
\end{abstract}

Keywords: discontinuous Galerkin, Nitsche's method, embedded grids, mesh locking

\section{Introduction}

Overlapping meshes can significantly simplify model development and provide a more generalized framework for model simulation. A common example is a continuum solid (foreground) mesh embedded in an Eulerian (background) fluid (Figure 1) but many different physics can be exploited in this context. Here, our proposed approach will be in the context of solid-to-solid interaction for simplicity but is extended to fluid-to-solid interaction in a sequel work.

${ }^{*}$ Corresponding author. Address: 7000 East Ave, Livermore, CA , 94550

Email addresses: sanders39@llnl.gov (Jessica Sanders), puso1@llnl.gov (Michael A. Puso)

${ }^{1}$ Methods Development Group, Lawrence Livermore National Laboratory, Livermore, CA 94550 


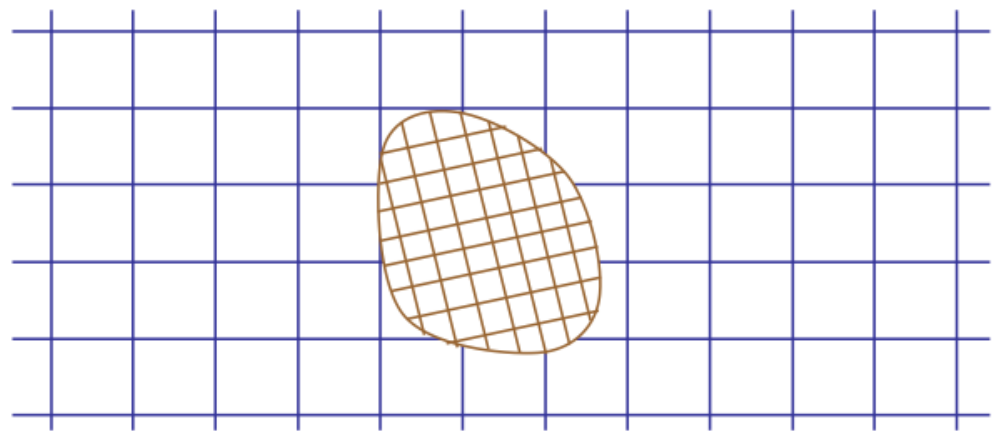

Figure 1: Embedded mesh approach. Background fluid in blue and foreground solid in brown.

The authors have previously investigated several techniques for embedded meshes in linear elasticity in [1]. In this work, it is shown that a standard mortar constraint approach (e.g., [3]) is prone to mesh locking, especially in the case of disparate mesh sizes and domain stiffness. A good exposition of the mesh locking phenomenon is presented in [4]. Methods that use an over-set grid are often applied in fluid mechanics (e.g., the Chimera method [5]) can avoid this mesh locking by building an auxiliary mesh using the background material. Penalty methods and Nitsche's method have also been studied for embedded meshes ([1] and [6] ). Applied appropriately, Nitsche's method was shown in [1] to enforce the two-body surface-to-volume constraints without mesh locking, and apparently with the correct rates of convergence, for linear elasticity.

Nitsche's method was originally proposed in [7] as a method for weakly imposing essential boundary condition for the finite element approximation of elliptical PDEs. A relationship to stabilized finite element methods was later established in [8], where it was shown that Nitsche's method could be derived for the stabilized Lagrange multiplier formulation of [9]. The method has witnessed a resurgence in recent years. It was also used in [10] and [11] for boundary condition enforcement in mesh-free methods, in [12] for domain decomposition purposes, and in [13] for contact, and in [14] and [15] for embedded interfaces ${ }^{2}$. Nitsche's method has posed some outstanding challenges for extension to large deformation, and other non-linear mechanics problems. In particular, the formulation for solid mechanics systems includes a stress variation operator (shown in Section 3, equation (11)) which is added to the statement of virtual work to assure symmetry and adjoint consistency of the virtual work. It has previously not been clear how to obtain a natural extension of the operator to a non-linear material law.

We address these problems by exploiting the well-documented parallels between Nitsche's method, interior penalty and discontinuous Galerkin (DG) methods. Discontinuous Galerkin (DG) methods have been used for the numerical solution of PDEs since their introduction

\footnotetext{
${ }^{2}$ Embedded interfaces in this context refer to surfaces that capture a jump of some field within a single mesh, as opposed to the embedding of two different meshes.
} 
in [16]. The class of methods has since been studied in many contexts, [e.g., 17, 18] and developed parallel to the similar class of methods known as interior penalty [e.g., 19, 20].

Elastic one-field problems with kinematic constraints, such as incompressibility, pose a well-known locking problem for conforming approximations. Locking in this context (which is similar in spirit to interface locking) motivated studies of DG methods for these elliptical classes of problems, along side other techniques such as reduced integration and assumed strain methods. DG methods avoid locking for incompressible elasticity through the relaxation of the assumptions of continuity at element boundaries (followed by subsequent weak enforcement to that continuity). The techniques in this chapter are largely motivated by the work of [21] and [17], which extend the ideas of the original DG methods as applied to linear elasticity by [18]. An excellent review of DG methods can be found in [2].

Whereas in standard finite element methods, continuity between elements is built into the discrete space, it must be enforced in DG methods in the variational form. The interior penalty approach is one way to apply these constraints. Its virtual work statement for linear elasticity is nearly form identical to Nitsche's method. The largest difference appears to be that "interior penalty" generally refers to the discrete variational forms used to enforce internal continuity for DG methods, whereas "Nitsche's method" generally refers to methods as applied to constraints and boundary conditions in a continuum sense. Many previous works acknowledge the fundamental connection between interior penalties, DG methods, and Nitsche's method, including [2], and the work of [22], which successfully blends the extended finite element method with DG methods for embedded discontinuities.

Derivation of interior penalty methods can be done with a discontinuous Galerkin derivative. For solid mechanics problems, this DG derivative relates a possible displacement jump in a solution field to an associated volumetric strain. Nitsche's method is not generally described with a DG derivative, but in this paper we show that the derivative may be adapted from its standard use for discrete spaces to our situation in which a displacement jump arises inherently from the definition of the problem. We show that for a linear system, the variational formulation resulting from using this operator is essentially equivalent to Nitsche's method prior to discretization.

Current foundations for discontinuous Galerkin methods in non-linear finite elasticity are presented in [23] and [24], based upon a natural extension of the linear work in [21] for incompressible elasticity. In these works, stability of the formulation is discussed via the problem of coercivity of the linearized elasticity problem. Though restrictions on the elastic moduli are shown to result in the desired coercivity properties, a complete case for overall stability of the formulation remains an open question.

We draw on the basic ideas in [24], in that we take advantage of their justification for extension of a DG derivative for a hyperelastic material. We adapt the concept of a DG derivative to the case in which the discontinuity is part of the problem definition, as opposed to discretely introduced, and apply it to both linear and non-linear elastic structural mechanics systems. The remainder of the document will be organized as follows: Section 2 introduces the problem formulation and governing equations for a two-body structural system with one body embedded in the other. Variational and discrete forms are presented 
in sections 3 and 4 . Section 5 reviews implementational details, and finally three numerical examples are presented in section 6 .

\section{Problem Formulation}

We consider the two domains shown in Figure $2, B^{1}$ and $B^{2}$, subject to finite deformation and forced to move in concert at their interface, $\Gamma_{*}$. Our formulation is general to cases of both complete (as shown) and partial overlap of the bodies. The coordinates of body $m$ in the material configuration are denoted as $\boldsymbol{X}^{(m)}$, and coordinates in the current configuration are denoted as $\boldsymbol{x}^{(m)}$. The deformation of the bodies are prescribed through the mapping $\boldsymbol{x}^{(m)}=\boldsymbol{\varphi}^{(m)}\left(\boldsymbol{X}^{(m)}\right)$.

The entire computational domain is denoted $\Omega$ and is the union of the background and foreground domains i.e., $\Omega=B^{1} \cup B^{2}$. Excluding $\Gamma_{*}$, the computational boundary can be divided into Dirichlet and Neumann parts on each body, $\Gamma_{d}^{(m)}$ and $\Gamma_{h}^{(m)}$, respectively.

For simplicity, we consider the case in which the materials are not subject to body loads, though we allow the possibility of surface loads, $\boldsymbol{T}^{(m)}$, and prescribed boundary displacements, $\overline{\boldsymbol{u}}^{(m)}$. The equations of motion are

$$
\begin{aligned}
\nabla_{0} \cdot \boldsymbol{P}^{(m)} & =0 & & \text { in } \Omega \\
\boldsymbol{u}^{(m)} & =\overline{\boldsymbol{u}}^{(m)} & & \text { on } \Gamma_{d}^{(m)} \\
\boldsymbol{P}^{(m)} \boldsymbol{n}^{(m)} & =\boldsymbol{T}^{(m)} & & \text { on } \Gamma_{h}^{(m)} \\
\boldsymbol{u}^{(1)} & =\boldsymbol{u}^{(2)} & & \text { on } \Gamma_{*} \\
\boldsymbol{P}^{(1)} \boldsymbol{n}^{(1)} & =-\boldsymbol{P}^{(2)} \boldsymbol{n}^{(2)} & & \text { on } \Gamma_{*} .
\end{aligned}
$$

For a hyperelastic deformation model, the first Piola-Kirchoff stress, $\boldsymbol{P}^{(m)}$, is obtained via direct differentiation of some stored energy function, $W^{(m)}\left(\boldsymbol{X}^{(m)}, \boldsymbol{F}^{(m)}\right)$, with respect to the deformation gradient, $\boldsymbol{F}^{(m)}$ :

$$
\boldsymbol{P}^{(m)}=\frac{\partial W^{(m)}}{\partial \boldsymbol{F}^{(m)}} .
$$

Equations (1d) and (1e) represent motion coupling through enforced continuity at the domains' shared boundary.

We also consider the elastic small deformation case in which stress is a linear function of strain, which is taken as the symmetric part of the displacement gradient, $\boldsymbol{\varepsilon}^{(m)}=\frac{1}{2}\left(\nabla \boldsymbol{u}^{(m)}+\nabla^{T} \boldsymbol{u}^{(m)}\right)$. Equation (1a) then reduces to $\nabla \cdot\left(\boldsymbol{C}^{(m)}: \boldsymbol{\varepsilon}^{(m)}\right)=0$. Here, $\boldsymbol{C}^{(m)}$ is the fourth order tensor of material properties for body $m$ according to Hooke's Law. 


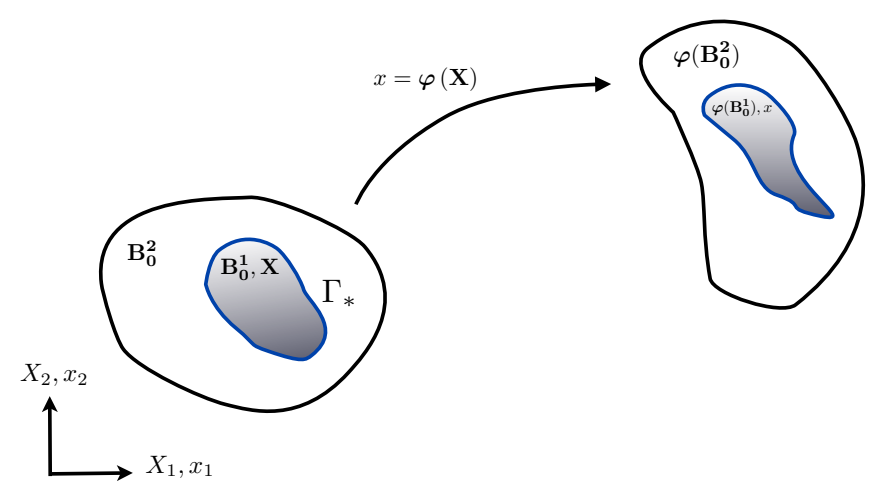

Figure 2: Two body problem in finite deformation

\section{Variational Forms}

For a traditional derivation of the finite element weak form associated with the equations of motion, we define a solution and weighting space, $\mathcal{S}^{(m)}$ and $\boldsymbol{V}^{(m)}$, consisting of displacements, $\boldsymbol{u}$, and their variations, $\delta \boldsymbol{u}$ :

$$
\begin{aligned}
\mathcal{S}^{(m)} & =\left\{\boldsymbol{u}^{(m)} \mid \boldsymbol{u}^{(m)} \in \boldsymbol{H}^{1}\left(B^{m}\right), \boldsymbol{u}^{(m)}=\overline{\boldsymbol{u}}^{(m)} \text { on } \Gamma_{d}^{(m)}\right\} \\
\boldsymbol{V}^{(m)} & =\left\{\delta \boldsymbol{u}^{(m)} \mid \delta \boldsymbol{u}^{(m)} \in \boldsymbol{H}^{1}\left(B^{m}\right), \delta \boldsymbol{u}^{(m)}=0 \text { on } \Gamma_{d}^{(m)}\right\} .
\end{aligned}
$$

The constrained variational problem is given by:

Find $\left(\boldsymbol{u}^{1}, \boldsymbol{u}^{2}\right) \in\left(\mathcal{S}^{1} \times \mathcal{S}^{2}\right)$ such that

$$
\begin{aligned}
\sum_{m} \int_{B_{0}^{(m)}}\left(\delta \boldsymbol{F}^{(m)}\right)^{T}: \boldsymbol{P}^{(m)} d B=\sum_{m} \int_{\partial B^{(m)}} \delta \boldsymbol{u}^{(m)} \cdot \boldsymbol{P}^{(m)} \boldsymbol{n}^{(m)} d \Gamma \\
\text { for all }\left(\delta \boldsymbol{u}^{1}, \delta \boldsymbol{u}^{2}\right) \in\left(\boldsymbol{V}^{1} \times \boldsymbol{V}^{2}\right) .
\end{aligned}
$$

The boundary terms on the right side can be divided into those evaluated over $\Gamma_{d}^{(m)}$, which vanish due to the definition of $\boldsymbol{V}$, those evaluated over $\Gamma_{h}^{(m)}$, in which we can make the substitution given by (1c), and those on the common boundary, $\Gamma_{*}$, These substitutions lead to the form:

Find $\left(\boldsymbol{u}^{1}, \boldsymbol{u}^{2}\right) \in\left(\mathcal{S}^{1} \times \mathcal{S}^{2}\right)$ such that

$$
\begin{array}{r}
\sum_{m} \int_{B_{0}^{(m)}}\left(\delta \boldsymbol{F}^{(m)}\right)^{T}: \boldsymbol{P}^{(m)} d B=\int_{\Gamma_{*}}[[\delta \boldsymbol{u}]] \cdot \hat{\boldsymbol{P}} \boldsymbol{n}^{(1)} d \Gamma+\sum_{m} \int_{\Gamma_{h}^{(m)}} \delta \boldsymbol{u}^{(m)} \cdot \boldsymbol{T}^{(m)} d \Gamma \\
\text { for all }\left(\delta \boldsymbol{u}^{1}, \delta \boldsymbol{u}^{2}\right) \in\left(\boldsymbol{V}^{1} \times \boldsymbol{V}^{2}\right) .
\end{array}
$$


Where the term $\hat{\boldsymbol{P}} \boldsymbol{n}^{(1)}$ indicates a developed contract traction on the shared interface. The jump in any field is denoted $[[\boldsymbol{a}]]=\boldsymbol{a}^{(1)}-\boldsymbol{a}^{(2)}$.

Standard mortar methods for this problem would devise a separate approximation space for the contact traction, $\hat{\boldsymbol{P}} \boldsymbol{n}^{(1)}$. In this altered approach we incorporate the displacement gap, $[[\boldsymbol{u}]]$, into the definition of the displacement gradient and show that this reproduces the appropriate constraint energy. This approach is motivated by discontinuous Galerkin methods. To define a displacement gradient augmented by the displacement jump, we make use of the lifting operators commonly used for discontinuous Galerkin style derivatives. The proposed operator, $\mathbf{R}$, converts a displacement jump, defined over a boundary, to a spatial gradient defined over an associated domain. It is defined through its action on a vector field $\boldsymbol{a}$ and a tensor field $\boldsymbol{b}$ via:

$$
\int_{\Omega} \mathbf{R}(\boldsymbol{a}): \boldsymbol{b} d \Omega=-\int_{\Gamma_{*}} \boldsymbol{a} \otimes \boldsymbol{n}^{(1)}: \boldsymbol{b}^{(2)} d \Gamma .
$$

We adapt the definition by choosing the $\boldsymbol{a}$ to be the displacement jump, $\boldsymbol{b}$ to be stress, and the domain of integration to coincide with $B^{2}$, which gives

$$
\begin{aligned}
\int_{B^{2}} \mathbf{R}([[\boldsymbol{u}]]): \boldsymbol{P} d B & =-\int_{\Gamma_{*}}[[\boldsymbol{u}]] \otimes \boldsymbol{n}^{(1)}: \boldsymbol{P} d \Gamma \\
& =-\int_{\Gamma_{*}}[[\boldsymbol{u}]] \cdot \boldsymbol{P} \boldsymbol{n}^{(1)} d \Gamma
\end{aligned}
$$

An integration domain of $B^{1}$ would also be possible, or a convex combination of integrals over each. We choose $B^{2}$ (the background) because it simplifies implementation, and was shown in numerical experimentation to be the most effective for the kind of stiff overlapping body problems for which we intend the method. The choice does affect the specifics of all of the following variational statements as well as implementation.

The operator $\mathbf{R}$ is tensor valued and defined over a volume, and we will need to chose a suitable basis and discrete expression for it. Section 4 will give more specifics into choosing and evaluating $\mathbf{R}$. A new gradient operator for the problem, based on both the traditional term $\boldsymbol{F}$ and the lifting operator, defined

$$
\boldsymbol{D}_{D G} \boldsymbol{u}=\boldsymbol{F}+\mathbf{R}([[\boldsymbol{u}]])
$$

is used in place of the standard displacement gradient for the compatible strains over the intersected background body. We consider the internal virtual work of body 2 (the background) and replace the gradient, $\boldsymbol{F}$ and the gradient variation, $\delta \boldsymbol{F}$, with their DG counterparts from 
(7), and expand the integral in the following way:

$$
\begin{aligned}
\int_{B^{2}}\left(\delta \boldsymbol{D}_{D G}^{(2)}\right)^{T}: \boldsymbol{P}^{(2)} d B & =\int_{B^{2}}\left(\delta \boldsymbol{F}^{(2)}\right)^{T}: \boldsymbol{P}^{(2)} d B+\int_{B^{2}} \mathbf{R}([[\delta \boldsymbol{u}]]): \boldsymbol{P}^{(2)} d B \\
& =\int_{B^{2}}\left(\delta \boldsymbol{F}^{(2)}\right)^{T}: \boldsymbol{P}^{(2)} d B-\int_{\Gamma_{*}}[[\delta \boldsymbol{u}]] \otimes \boldsymbol{n}^{(1)}: \boldsymbol{P}^{(2)} d \Gamma
\end{aligned}
$$

The last term in (8b) gives the term representing constraint energy that was unknown - the second term in equation (4). By replacing the operator $\boldsymbol{F}$ with $\boldsymbol{D}_{D G}$, we effectively move the term representing constraint energy to the left side of the equation and incorporate it into the body integral. Our variational form becomes

Find $\left(\boldsymbol{u}^{1}, \boldsymbol{u}^{2}\right) \in\left(\mathcal{S}^{1} \times \mathcal{S}^{2}\right)$ such that

$$
\begin{array}{r}
\int_{B^{1}}\left(\delta \boldsymbol{F}^{(1)}\right)^{T}: \boldsymbol{P}^{(1)} d B+\int_{B^{2}}\left(\delta \boldsymbol{D}_{D G}^{(2)}\right)^{T}: \boldsymbol{P}^{(2)} d B=\sum_{m} \int_{\Gamma_{h}^{(m)}} \delta \boldsymbol{u}^{(m)} \cdot \boldsymbol{T}^{(m)} d \Gamma \\
\text { for all }\left(\delta \boldsymbol{u}^{1}, \delta \boldsymbol{u}^{2}\right) \in\left(\boldsymbol{V}^{1} \times \boldsymbol{V}^{2}\right) .
\end{array}
$$

We can see the relationship to Nitsche's method by investigating the linear case, in which (9) becomes

Find $\left(\boldsymbol{u}^{1}, \boldsymbol{u}^{2}\right) \in\left(\mathcal{S}^{1} \times \mathcal{S}^{2}\right)$

$$
\begin{array}{r}
\int_{B^{1}} \delta \boldsymbol{\varepsilon}^{(1)}: \boldsymbol{C}^{(1)}: \boldsymbol{\varepsilon}^{(1)} d B+\int_{B^{2}} \delta \boldsymbol{D}_{D G}^{(2)}: \boldsymbol{C}^{(2)}: \boldsymbol{D}_{D G}^{(2)} d B=\sum_{m} \int_{\Gamma_{h}^{(m)}} \delta \boldsymbol{u}^{(m)} \cdot \boldsymbol{T}^{(m)} d \Gamma \\
\text { for all }\left(\delta \boldsymbol{u}^{1}, \delta \boldsymbol{u}^{2}\right) \in\left(\boldsymbol{V}^{1} \times \boldsymbol{V}^{2}\right) .
\end{array}
$$

Equation (10) can be expanded and re-written using the definition of $\mathbf{R}$ :

Find $\left(\boldsymbol{u}^{1}, \boldsymbol{u}^{2}\right) \in\left(\mathcal{S}^{1} \times \mathcal{S}^{2}\right)$

$$
\begin{gathered}
\sum_{m} \int_{B^{(m)}} \delta \boldsymbol{\varepsilon}^{(m)}: \boldsymbol{C}^{(m)}: \boldsymbol{\varepsilon}^{(m)} d \Omega-\int_{\Gamma_{*}}[[\delta \boldsymbol{u}]] \otimes \boldsymbol{n}^{(1)}: \boldsymbol{C}^{(2)}: \boldsymbol{\varepsilon}^{(2)} d \Gamma \\
-\int_{\Gamma_{*}}[[\boldsymbol{u}]] \otimes \boldsymbol{n}^{(1)}: \boldsymbol{C}^{(2)}: \delta \boldsymbol{\varepsilon}^{(2)} d \Gamma+\int_{B^{2}} \mathbf{R}([[\delta \boldsymbol{u}]]): \boldsymbol{C}^{(2)}: \mathbf{R}([[\boldsymbol{u}]]) d B= \\
\qquad \sum_{m} \int_{\Gamma_{h}^{(m)}} \delta \boldsymbol{u} \cdot \boldsymbol{T}^{(m)} d \Gamma \\
\quad \text { for all }\left(\delta \boldsymbol{u}^{1}, \delta \boldsymbol{u}^{2}\right) \in\left(\boldsymbol{V}^{1} \times \boldsymbol{V}^{2}\right) .
\end{gathered}
$$

If the integral $\int_{B^{2}} \mathbf{R}([[\delta \boldsymbol{u}]]): \boldsymbol{C}: \mathbf{R}([[\boldsymbol{u}]]) d B$ were replaced with a standard penalty term like $\alpha \int_{\Omega}[[\boldsymbol{u}]][[\delta \boldsymbol{u}]] d \Omega$, equation (11) would be identical to a Nitsche based approached to the constrained two body problem. Equation (9) can then be seen as the natural extension 
of Nitsche's method to a nonlinear elastic embedded mesh system.

The penalty style term in Nitsche's method has the function of stabilizing the problem, which would otherwise lose its coercivity (and discrete positive definiteness) due to the subtracted integrals. The stabilization term, $\alpha$, must be appropriately chosen. The final integral in (11) appears to act in the same function, but without a necessary choice of stabilization coefficient. It was shown in [2], however, that for traditional DG applications, the formulation is not unconditionally stable, meaning that it may be necessary to add an extra stabilization term. None of our examples in linear elasticity appeared to require this extra stabilization, though some of the nonlinear examples benefited from a small extra penalty term.

\section{Discretization}

The bodies are meshed independently, with a discretization mismatch allowed to occur at $\Gamma_{*}$. Finite dimensional subspaces in this case are:

$$
\begin{gathered}
\mathcal{S}^{(m)^{h}}=\left\{\boldsymbol{u}^{(m)^{h}} \mid \boldsymbol{u}^{(m)^{h}} \in C^{0}\left(B^{m}\right), \boldsymbol{u}=\overline{\boldsymbol{\varphi}} \text { on } \Gamma_{d}^{(m)}\right\} \\
\boldsymbol{V}^{(m)^{h}}=\left\{\delta \boldsymbol{u}^{(m)^{h}} \mid \delta \boldsymbol{u}^{(m)^{h}} \in C^{0}\left(B^{m}\right), \delta \boldsymbol{u}=0 \text { on } \Gamma_{d}^{(m)}\right\} .
\end{gathered}
$$

Figure 3 shows the overlapping body superposed over the background discretized body 2 . For simplicity, the overlapping elements are not shown.

All elements of body 1 contribute to the discrete stiffness of the system. Body 2 contains three categories of elements: (1) those that are submerged (void) and contribute no stiffness, (2) those that are complete, and (3) cut elements which contribute partial stiffness. These cut elements use the discrete DG strain:

$$
\boldsymbol{D}_{D G} \boldsymbol{u}^{h}=\mathbf{1}+\frac{\partial N_{A}}{\partial \boldsymbol{X}} \boldsymbol{d}_{A}+\mathbf{R}\left(\left[\left[\boldsymbol{u}^{h}\right]\right]\right)
$$

or

$$
D_{D G}=\delta_{i I}+\frac{\partial N_{A}}{\partial X_{I}} d_{A i}+R_{i I}^{h}\left(\left[\left[u_{i}^{h}\right]\right]\right) .
$$

Note that when using indicial notation, we use capital letters for quantities measured in material coordinates, and lower case letters for spatial coordinates.

In a modified DG element we need to be able to form a discretization of the term $\mathbf{R}\left(\left[\left[\boldsymbol{u}^{h}\right]\right]\right)$. This is a local calculation which involves choosing a basis for $\mathbf{R}$. Here we present two approaches, the first taken in [24], and a second which was implemneted for the subsequent numerical results. For the following development, a section of $\Gamma_{*}$ that overlaps a specific element, $e$, is called $\Gamma_{e}$. For convenience, we introduce a special vector of displacements,

$$
\boldsymbol{d}^{g}=\left\{\begin{array}{c}
\boldsymbol{d}_{e}^{(1)} \\
\boldsymbol{d}_{e}^{(2)}
\end{array}\right\}
$$




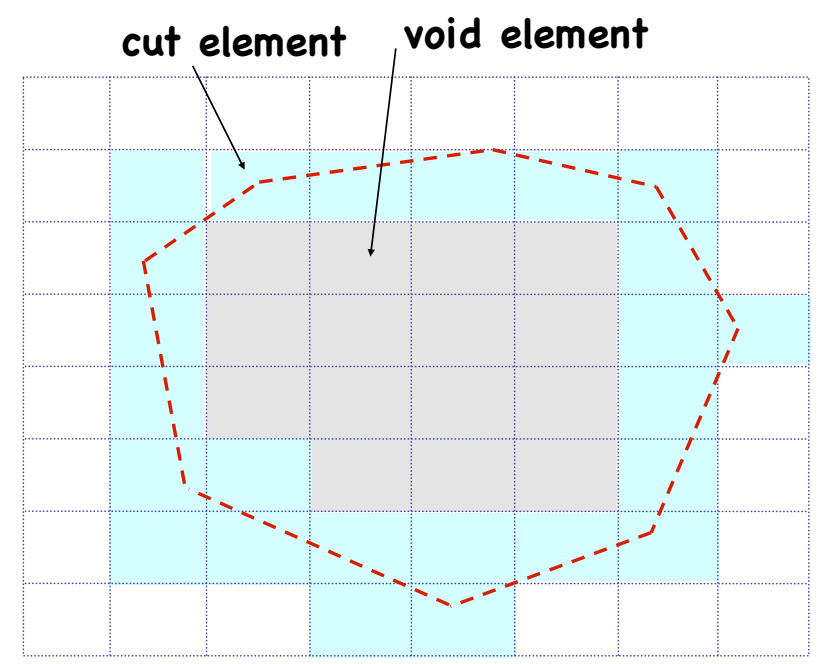

Figure 3: The background grid divided into traditional (white), void (gray) and DG elements (teal). Foreground boundary is shown with a dotted line.

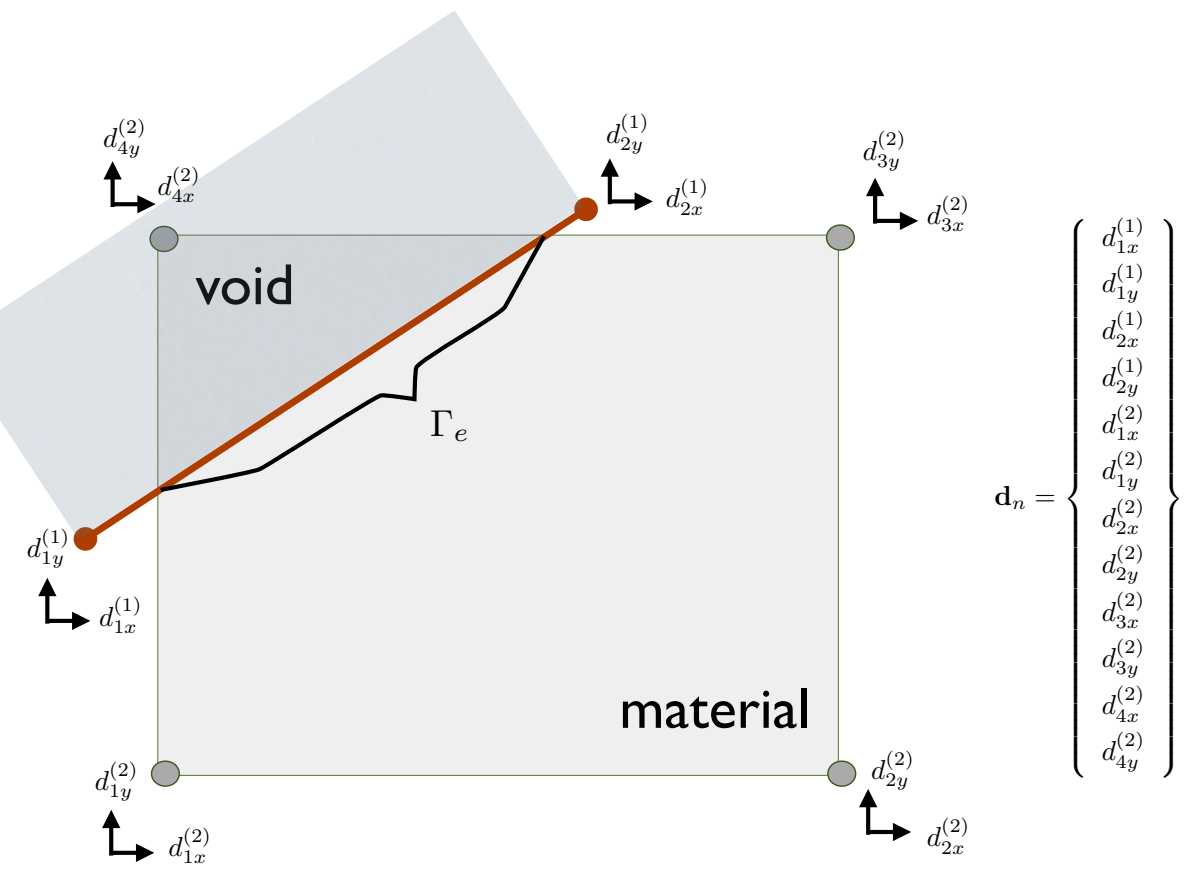

Figure 4: DG element with associated degrees of freedom 
corresponding to all of the displacement degrees of freedom involved in the discrete gap function over $\Gamma_{*}^{e}$. Figure 4 shows an example. Assuming that $\mathbf{R}$ has support functions $\boldsymbol{N}_{B}$ over the entire area of a DG element, we can write

$$
\mathbf{R}\left(\left[\left[\boldsymbol{u}^{h}\right]\right]\right)=\boldsymbol{N}_{B}\left[\mathbf{R}_{B}\right] \boldsymbol{d}^{g}
$$

where $B$ indexes and is summed over the chosen supports of $\mathbf{R}$. For clarity it is worth presenting both vector and indicial notation, such that

$$
R_{i J}([[\boldsymbol{u}]])=N_{B}\left[R_{B J}\right]\left[\left[u_{i}\right]\right]=N_{B}\left[R_{B A J}\right] d_{A i}^{g}
$$

where $B$ is indexed over all of the elements involved in the gap function as defined over $\Gamma_{*}^{e}$. We solve for $\left[R_{B A J}\right]$ through a discrete form of the equation that defines $\mathbf{R}$. Equation (6b), written over a single element, $e$, is

$$
\int_{B_{e}} \mathbf{R}([[\boldsymbol{u}]]): \boldsymbol{P} d B=-\int_{\Gamma_{e}}\left([[\boldsymbol{u}]] \otimes \boldsymbol{n}^{(1)}\right): \boldsymbol{P} d \Gamma
$$

or,

$$
\int_{B_{e}} R_{i J}([[\boldsymbol{u}]]) P_{i J} d B=-\int_{\Gamma_{e}}\left[\left[u_{i}\right]\right] P_{i J} n_{J}^{(1)} d \Gamma .
$$

By substituting (16) into (17), assuming linear $N_{B}$, using the discrete form of $\boldsymbol{u}$, and acknowledging arbitrary $P_{i J}$, we can form a discrete system that can be solved for the coefficients of $\left[R_{B A J}\right]$. This was the approach of [24]. In the proposed approach, in lieu of solving a linear system, we let $\mathbf{R}$ be constant, such that we get the expression

$$
R_{i J}([[\boldsymbol{u}]]) \int_{B_{e}^{2}} d B=-\int_{\Gamma_{e}}\left[\left[u_{i}\right]\right] n_{J}^{(1)} d \Gamma,
$$

or

$$
R_{i J}([[\boldsymbol{u}]])=-\frac{1}{V_{E}} \int_{B_{E}}\left(N_{A}^{(1)}-N_{A}^{(2)}\right) n_{J} d_{A i}^{g} d \Omega,
$$

where $V_{E}$ is the volume, or area, of the material region of the cut element, and $n_{J}$ is the outward normal, in material coordinates, of $\Gamma_{*}$. The vector $d_{A i}^{g}$ is a set of nodal degrees of freedom associated with the support of the gap function. The volume integrals are only performed over the material, or uncovered area, meaning that $V_{E}$ is the area of the material section of the element.

It should be noted that the constant $\mathbf{R}$ can lead to rigid body rotation mode for a single foreground/background element pairing (See Figure (5)). We typically do not expect the mode to propagate for more than one element.

Once the virtual work statements have been discretized, Nitsche's method and the DG derivative method can no longer be seen as equivalent, due to the necessity of the discrete space approximation of the lifting function. 
Un-deformed elements

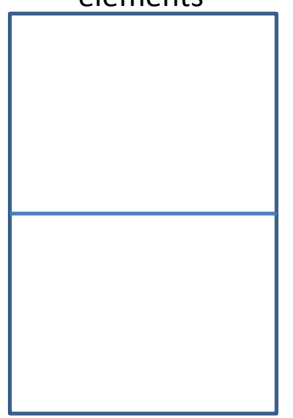

Possible deformation mode

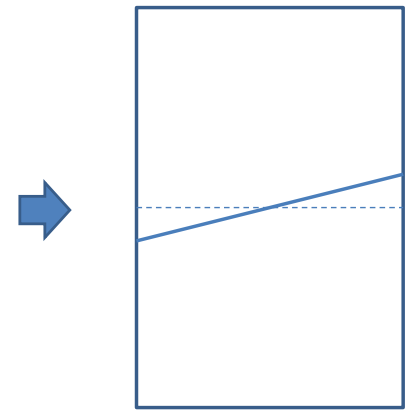

Figure 5: A rotational displacement jump mode may be transparent to the constant strain lifting operator

\section{Linear and non-linear implementational}

For a linear system the strain in a DG element appears as

$$
\boldsymbol{D}_{D G}=\boldsymbol{\varepsilon}(\boldsymbol{u})+\mathbf{R}([[\boldsymbol{u}]])=\boldsymbol{B d}+\boldsymbol{B}^{M} \boldsymbol{d}^{g},
$$

where $\boldsymbol{B}$ and $\boldsymbol{d}$ are the standard B matrix and element displacement vector and

$$
\begin{aligned}
& \boldsymbol{B}^{M}=-\left[\boldsymbol{B}_{1}^{M}, \boldsymbol{B}_{2}^{M}, \quad-\boldsymbol{B}_{3}^{M}, \ldots-\boldsymbol{B}_{n_{g}}^{M}\right], \\
& \boldsymbol{B}_{a}^{M}=\frac{1}{V_{e}}\left[\begin{array}{cc}
\int_{\Gamma_{*}^{e}} N_{a} n_{1} & 0 \\
0 & \int_{\Gamma_{*}^{e}} N_{a} n_{2} \\
\int_{\Gamma_{*}^{e}} N_{a} n_{2} & \int_{\Gamma_{*}^{e}} N_{a} n_{1}
\end{array}\right] .
\end{aligned}
$$

Here $n_{g}$ is the number of nodes involved in the special element displacement vector $\boldsymbol{d}^{g}$. The final expression for a modified element stiffness is

$$
\boldsymbol{k}^{e}=\int_{B_{e}}\left(\boldsymbol{B}+\boldsymbol{B}^{M}\right)^{T} \boldsymbol{D}\left(\boldsymbol{B}+\boldsymbol{B}^{M}\right) d B_{e},
$$

which must be assembled using a local ID array taking into account all of the degrees of freedom in $\boldsymbol{d}$ and $\boldsymbol{d}^{g}$.

For the non-linear system discretize the more convenient (and equivalent) variational 
form given by:

Find $\boldsymbol{u} \in \mathcal{S}^{1}$ such that

$$
\int_{\Omega} \delta \boldsymbol{E}: \boldsymbol{S} d \Omega=\int_{\Gamma_{h}^{(m)}} \delta \boldsymbol{u} \cdot \boldsymbol{T}^{(m)} d \Gamma
$$

for all $\delta \boldsymbol{u} \in \boldsymbol{V}$.

where $\boldsymbol{E}$ is the Green strain tensor and $\boldsymbol{S}$ is the second Piola-Kirchoff stress, both functionally dependent on $\boldsymbol{D}_{D G}$. The variation of strain in DG elements becomes

$$
\delta E_{I J}^{D G}=\frac{1}{2}\left(\frac{\partial \delta u_{i}}{\partial X_{I}}\left(F_{i I}+R_{i I}\right)+\frac{\partial \delta u_{i}}{\partial X_{J}}\left(F_{i J}+R_{i J}\right)\right) .
$$

In 2D Voigt vector notation this is

$$
\begin{aligned}
\delta \boldsymbol{E}^{D G} & \left.=\left\{\begin{array}{c}
F_{i 1} \delta u_{i, 1} \\
F_{i 2} \delta u_{i, 2} \\
F_{i 1} \delta u_{i, 2}+F_{i 2} \delta u_{i, 1}
\end{array}\right\}+\left\{\begin{array}{c}
R_{i 1} \delta u_{i, 1} \\
R_{i 2} \delta u_{i, 2} \\
R_{i 1} \delta u_{i, 2}+R_{i 2} \delta u_{i, 1}
\end{array}\right\} \text { (sum over } i\right), \\
& =\boldsymbol{B} \delta \boldsymbol{u}+\boldsymbol{B}^{M} \delta \boldsymbol{u}^{g}
\end{aligned}
$$

where $\boldsymbol{B}$ is the standard non-linear displacement gradient matrix, and

$$
\begin{gathered}
\boldsymbol{B}^{M}=-\left[\boldsymbol{B}_{1}^{M} \boldsymbol{B}_{2}^{M} \boldsymbol{B}_{3}^{M} \ldots \boldsymbol{B}_{n_{g}}^{M}\right] \\
\boldsymbol{B}_{A}^{M}=\left\{\begin{array}{cc}
R_{11} N_{A, 1} & R_{21} N_{A, 1} \\
R_{12} N_{A, 2} & R_{22} N_{A, 2} \\
R_{11} N_{A, 2}+R_{12} N_{A, 2} & R_{21} N_{A, 2}+R_{21} N_{A, 1}
\end{array}\right\} .
\end{gathered}
$$

Local evaluation of the internal forces in DG elements gives

$$
\boldsymbol{f}_{D G}^{i n t, e, m}(\boldsymbol{d})=\int_{B_{e}^{m}}\left(\boldsymbol{B}+\boldsymbol{B}^{M}\right)^{T} \boldsymbol{S}_{D G} d B_{e}
$$

The consistent tangent associated with the linearization of this term for a Newton-Raphson iteration is:

$$
\frac{\partial \boldsymbol{f}^{i n t}(\boldsymbol{d})}{\partial \boldsymbol{d}}=\int_{B_{e}}\left(\boldsymbol{B}+\boldsymbol{B}^{M}\right)^{T} \frac{\partial \boldsymbol{S}}{\partial \boldsymbol{E}}\left(\boldsymbol{B}+\boldsymbol{B}^{M}\right) d B_{e}+\int_{B_{e}} \frac{\partial\left(\boldsymbol{B}+\boldsymbol{B}^{M}\right)}{\partial \boldsymbol{d}} \boldsymbol{S} d B_{e}
$$

In the following examples, integration of the DG elements is done with Gauss quadrature over sub-triangles. Standard finite element assembly of element internal forces and tangent stiffness applies, and the global problem is solved with Newton-Raphson iteration. 


\section{Numerical Examples}

For three numerical examples, we compare a DG derivative approach to penalty and mortar constraint approaches for the same problem. For a description of penalty and mortar approaches to the embedded problem see [1].

For finite deformation problems, we use a standard Neo-Hookean hyperelastic behavioral law, based on the first invariant of strain, $I$, and the deformation jacobian, $J$, given by:

$$
W=\frac{1}{2} \mu(I-3-2 \ln J)+\frac{\lambda}{4}\left(J^{2}-1\right)-\frac{\lambda}{2} J .
$$

\subsection{Non-linear bending}

We construct a rectangular $[1 \times 1.5]$ background domain, with the origin at the center of the left boundary, and embed a smaller $[1 \times 0.625]$ rectangular body, in left portion, so that the boundaries coincide on three surfaces, and continuity only needs to be enforced on a vertical surface at $x=0.625$. The crosshatch mesh is constructed to represent a "worst case scenario" for the locking phenomenon, with the constrained surface cutting diagonally across the background mesh. Loading is a linear distribution of normal forces on the left and right faces. The embedded domain is both more finely meshed and comparatively stiff than the background grid $\left(E_{1}=50000, E_{2}=50, \nu_{1}=\nu_{2}=0.3\right)$. Finite deformations and the Neo-Hookean material law are considered.

Figure 6 shows a problem setup and a refined FEA solution with no embedded mesh. For the sake of rendering, the gridding is not shown. Figure 7 shows a mortar solution, both in terms of $\mathrm{x}$-directional stresses and interface traction, where it is seen that large oscillations in stress are encountered at the interface. Results for the DG derivative formulation are shown in Figure 8 and appear qualitatively improved. Convergence rates to a fine conforming mesh are given in Figures 9 and 10 for mortar methods, a penalty method, and the DG derivative method. The mortar method does not converge in the energy norm, and the penalty method does not converge optimally in the L2 norm of displacements, but the DG derivative method converges optimally or nearly optimally for both. Both the penalty method and the DG derivative method have slightly less than optimal rates in the energy norm. This may be a result of the non-linearities of the geometries and comparison to the imperfect finite element solution. Figure 11 is the energy convergence results for the equivalent linear problem, which in fact do converge optimally.

\subsection{Linear multiple inclusions}

A second problem, this time representing a small deformation linear system, is given in Figure 12. A conforming FEA solution for y-directional stresses is shown in Figure 13. Embedded meshes with a mortar constraint, and the DG derivative are shown in Figures 14 and 15, respectively. Convergence properties in the error norms for the three methods, as well as a standard Nitsche's method, are given in Figure 16. In both norms, convergence rates are better for the DG derivative method than for any of the other three. Results are shown with zero extra stabilization for the method, meaning that there is also no extra choice or scaling of a stability parameter. 


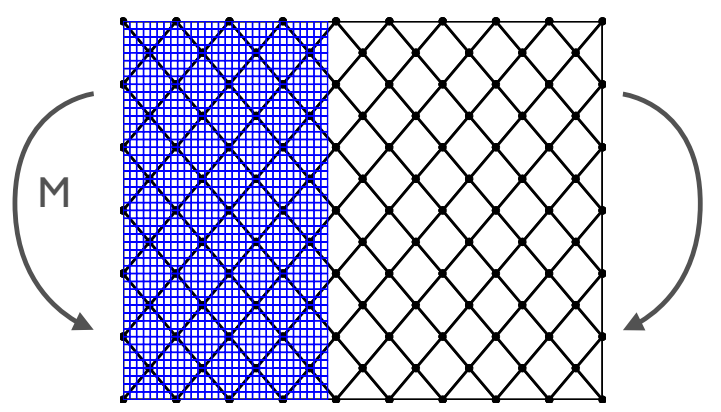

(a)

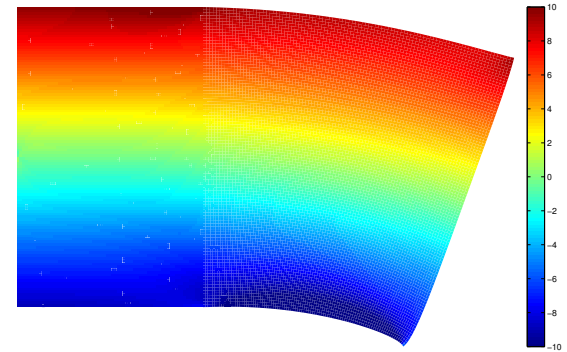

(b)

Figure 6: (a) Problem setup (b) Converged conforming finite element solution for non-linear bending

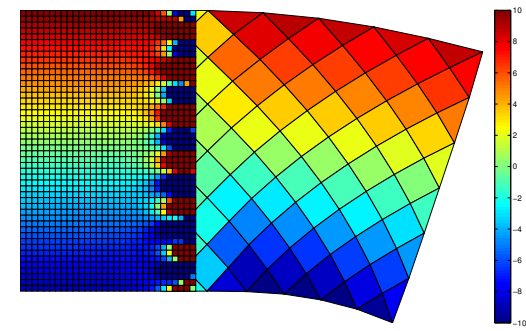

(a)

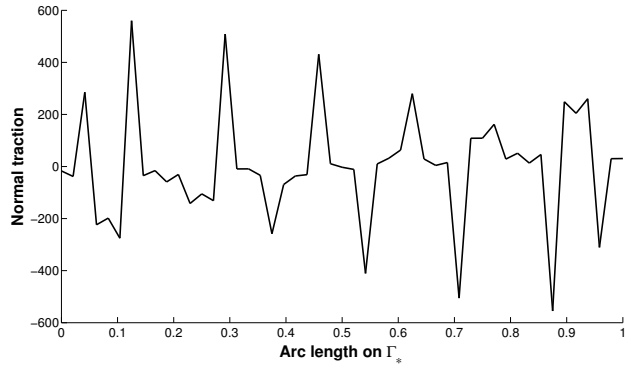

(b)

Figure 7: Mortar solutions for non-linear bending: (a) Cauchy stresses in x, (b) surface traction

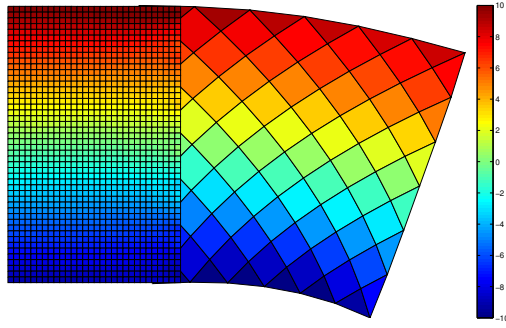

(a)

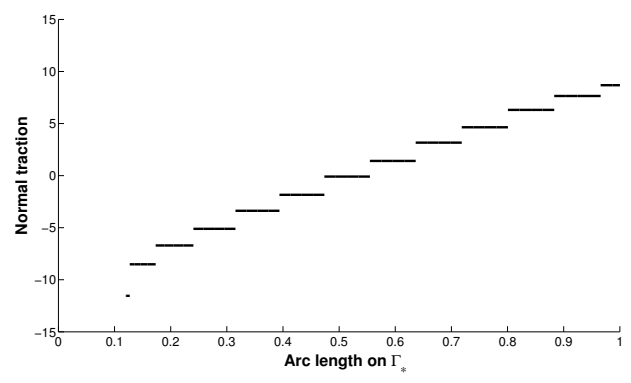

(b)

Figure 8: DG solutions for non-linear bending: (a) Cauchy stresses in $\mathrm{x}$, (b) surface traction 


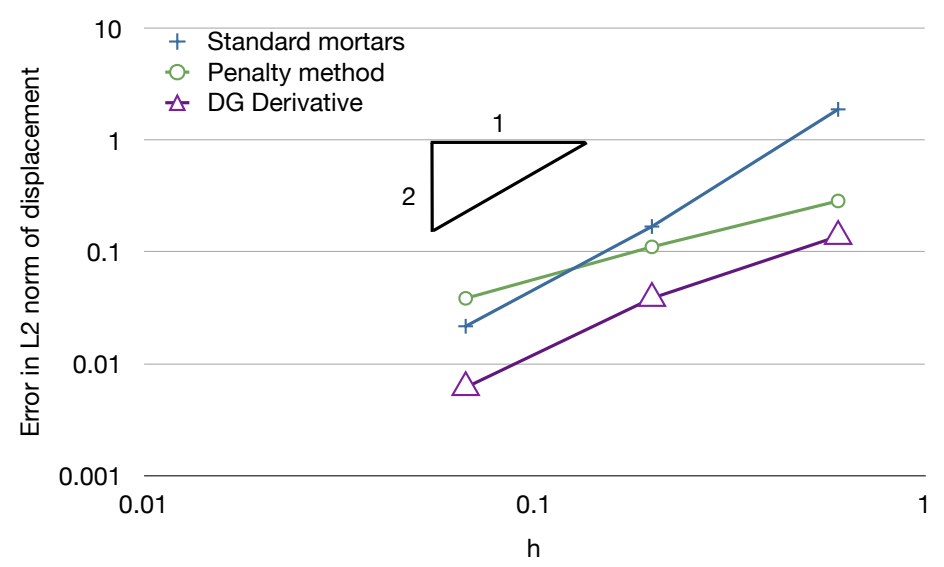

Figure 9: Spatial convergence for L2 norm in displacement for non-linear bending

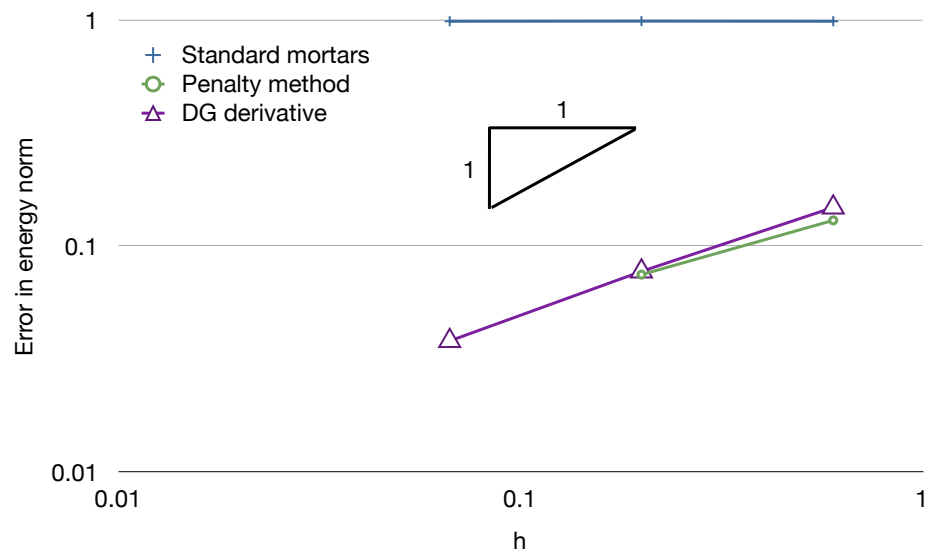

Figure 10: Spatial convergence for energy norm in displacement for non-linear bending 


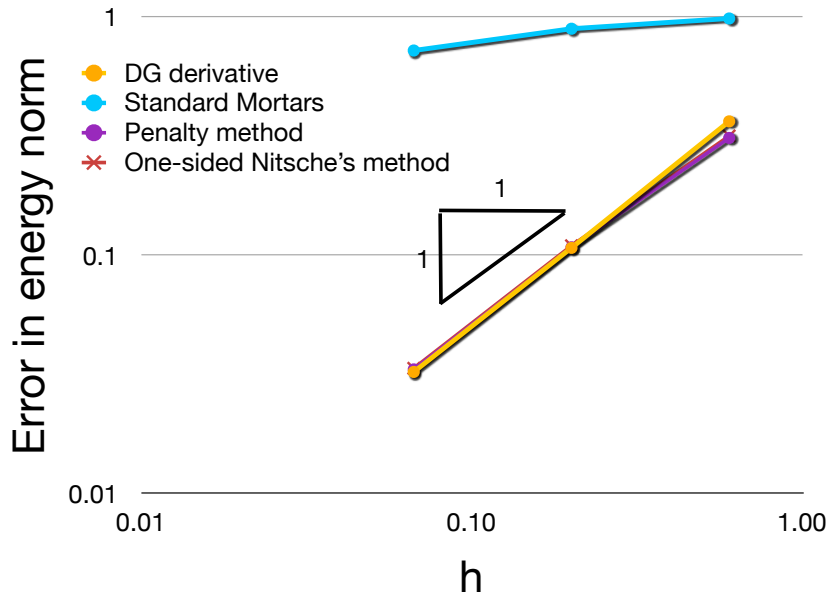

Figure 11: Spatial convergence for energy norm in displacement for linear bending

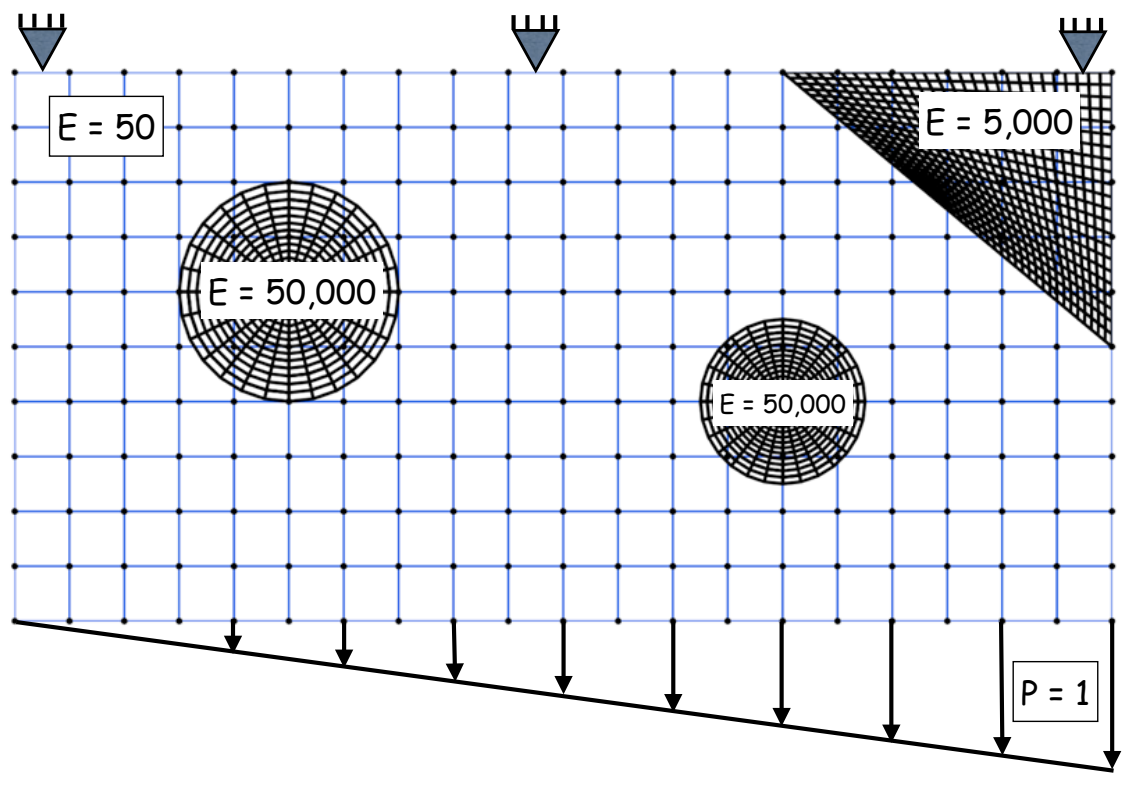

Figure 12: Setup for a multiple inclusion problem; all inclusions are stiff. 


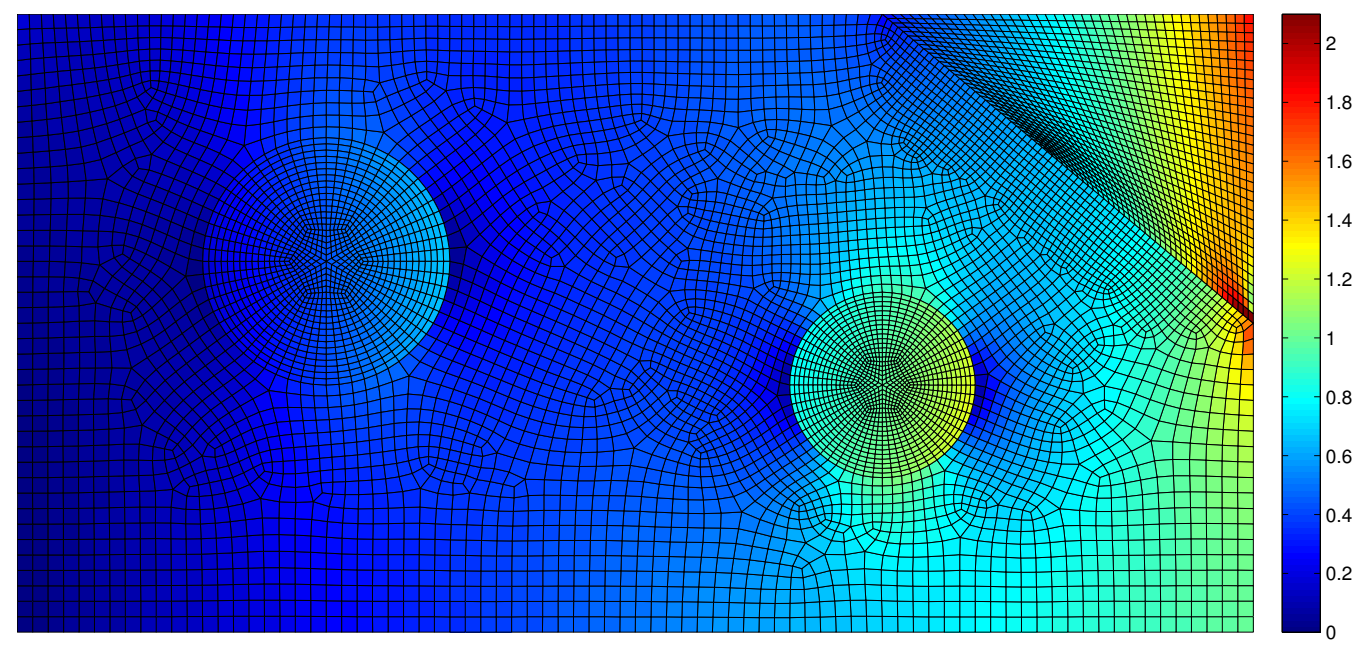

Figure 13: Reference solution (y-directional stresses) with a conforming finite element grid for stiff inclusion example

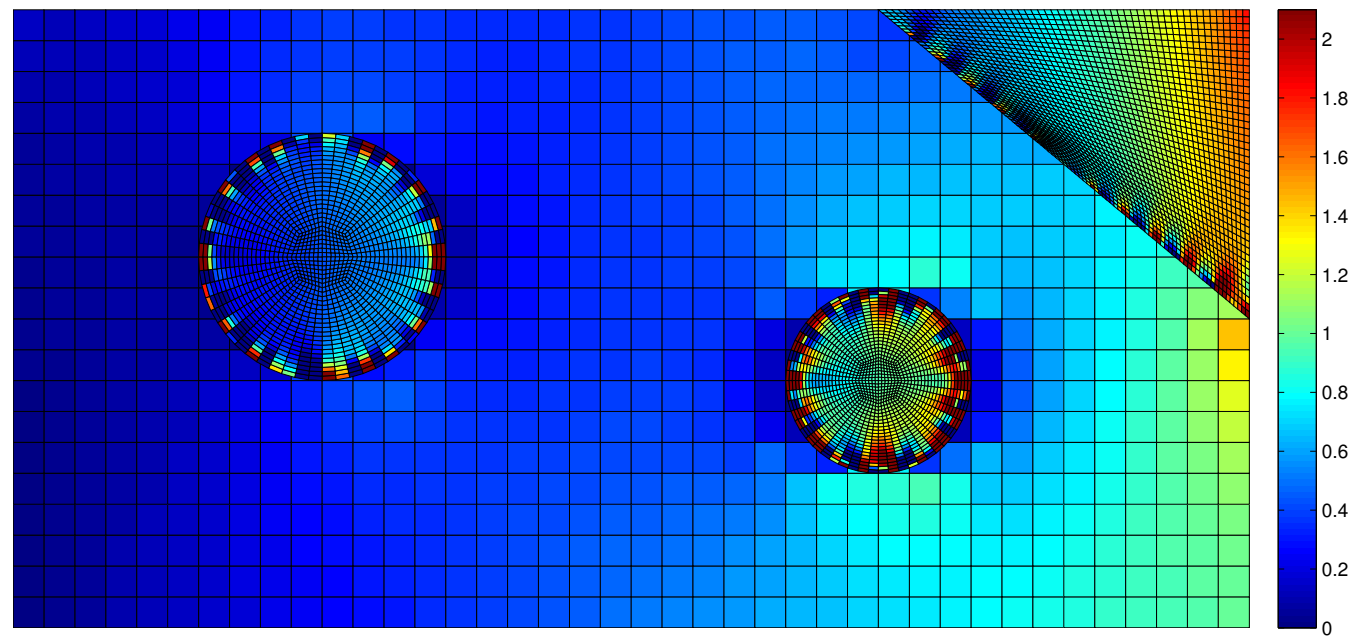

Figure 14: Mortar solution (y-directional stresses) for stiff inclusion example 


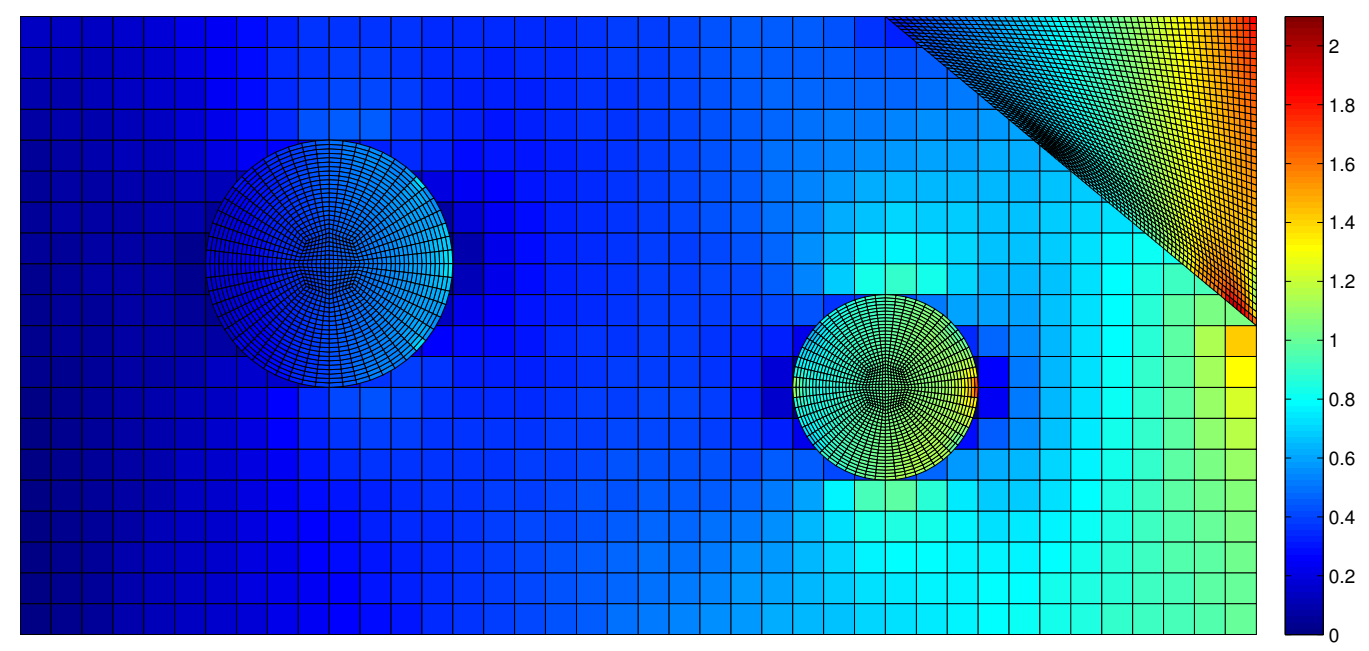

Figure 15: DG derivative solution (y-directional stresses) for stiff inclusion example

\subsection{Non-linear conveyor belt}

The final example is a cross-sectional plane strain simulation of loading on a reinforced conveyor belt, again with finite deformations and a Neo-Hookean material. These kinds of systems are used for heavy duty industrial applications such as mining. Steel cords are generally used as the reinforcement for a rubber belt. Both materials can be reasonably modeled with a Neo-Hookean constitutive model in the elastic regime, although their overall strength and compressibility are very different. A setup is shown in Figure 17. The rubber belt is modeled with a effective stiffness of $E=0.1$ and Poisson's ratio of $\nu=0.45$. The steel inclusions are modeled with a stiffness of $E=200$ and $\nu=0.3$. The right side of the problem is considered a symmetry plane, and we apply a pressure loading. The loading is deliberately larger than would be physically reasonable for industrial applications, so as to allow us to observe very large deformations of the materials. A contour of bending stresses for a conforming solution is shown in Figure 18. Though overall displacement seems reasonable in the mortar solution (Figure 19), it is clear that the stress values in the steel are nonphysical - this is due to the stiffness difference between the materials. The relative mesh densities were allowed to be similar in this case, to demonstrate that only one of our two important parameters - relative mesh density and relative stiffness, can be enough to produce a locking effect. The solution due to the DG method returns a reasonable approximation of the stress field in comparison to the conforming solution (Figure 20). 


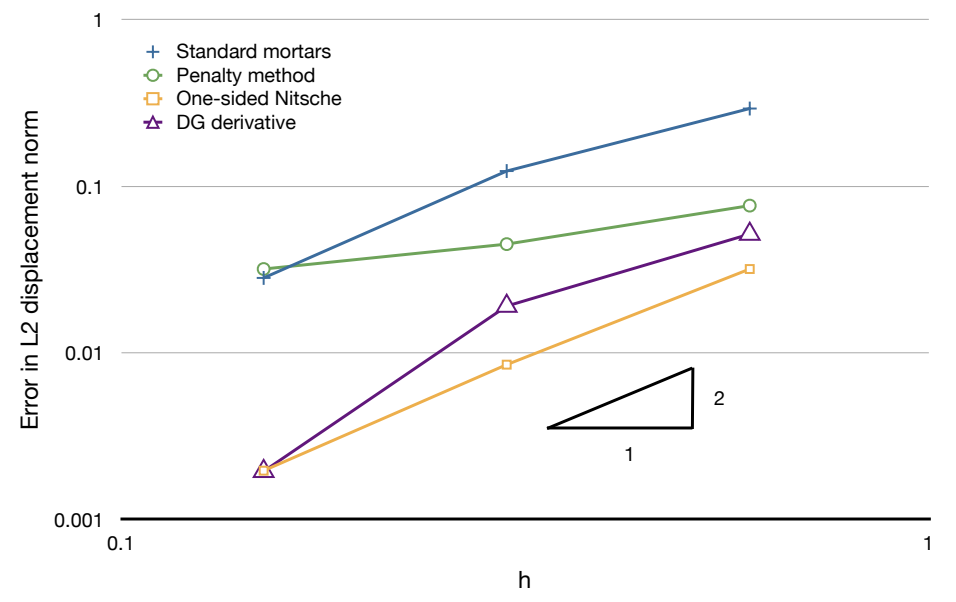

(a)

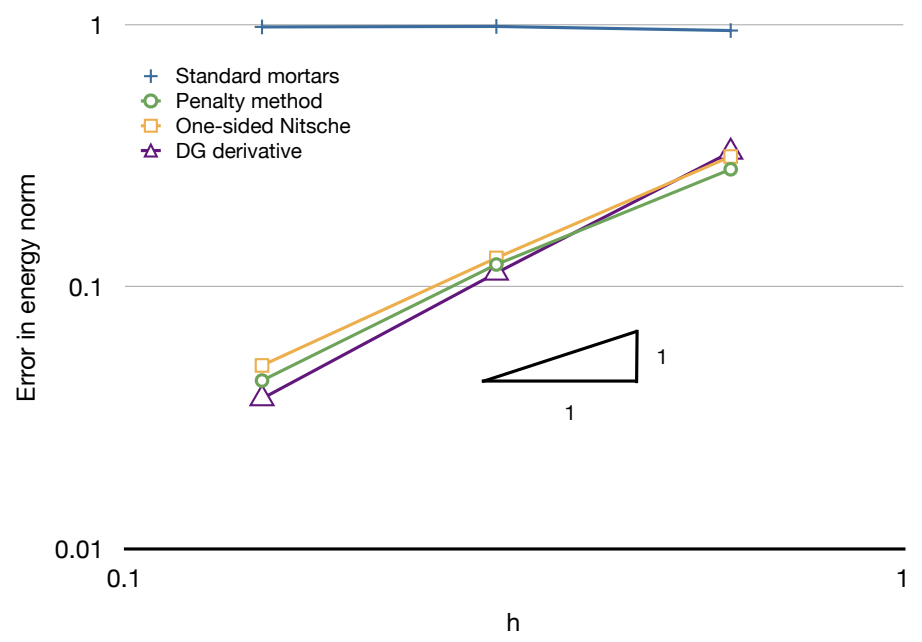

(b)

Figure 16: Spatial convergence to a fine conforming mesh for stiff inclusion problem

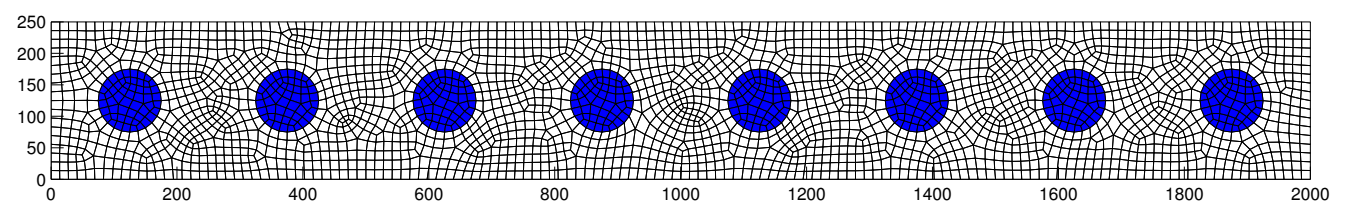

Figure 17: Setup for reinforced conveyor belt problem 


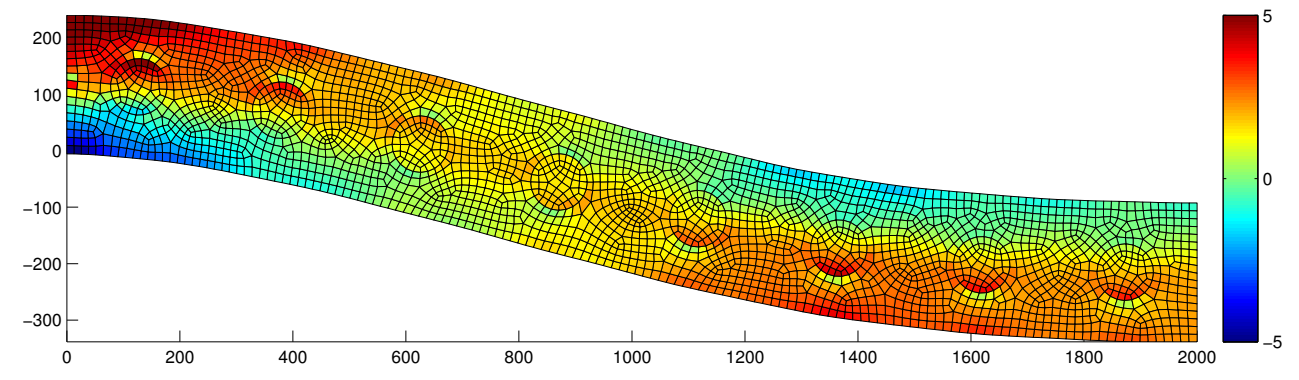

Figure 18: Conforming solution for reinforced conveyor belt problem (bending stresses)

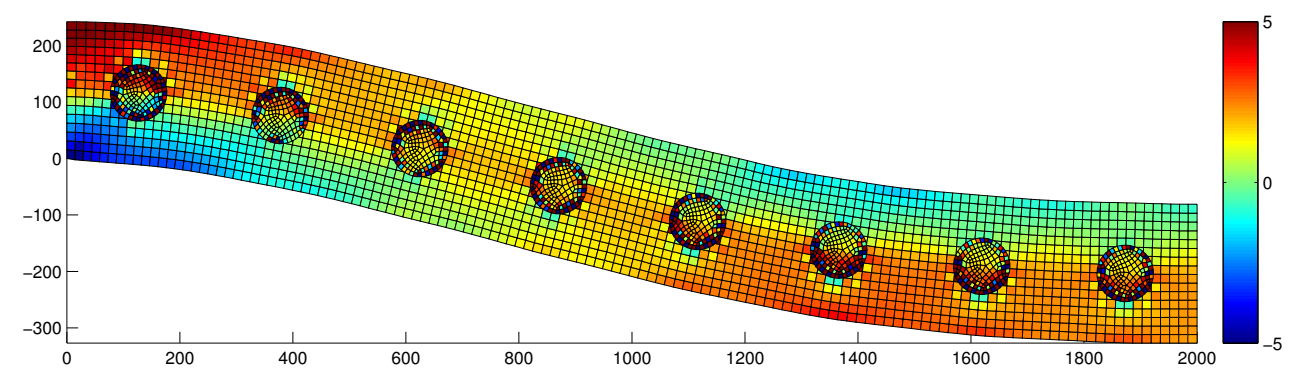

Figure 19: Mortar solution for reinforced conveyor belt problem (bending stresses)

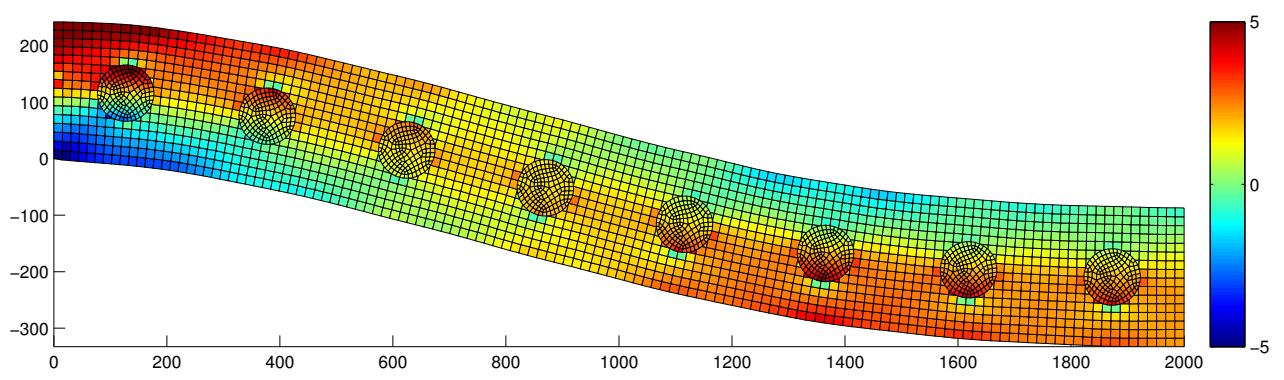

Figure 20: DG derivative solution for reinforced conveyor belt problem (bending stresses) 


\section{Conclusions}

In this paper, the stability of embedded finite element meshes for structural mechanics problems was analyzed. In the case of structural elasticity, traditional methods for enforcing continuity constraints at embedded boundaries have been shown to have either stability or consistency problems. In particular, mortar methods, which are standard for treatment of conformally meshed interfaces, can result in over-constraint and locking behavior, which causes a loss of energy convergence and unstable interfacial fluxes. Penalty methods tend to behave better than mortar methods for energy considerations, but failed to sufficiently enforce continuity constraints, resulting in loss of optimality in the displacement norms. Nitsche's method for weak constraint has been shown to be capable of restoring convergence optimality and increasing overall accuracy of the solution in these cases. The previous major disadvantage of Nitsche's method was that its extension to non-linear material behavior was unclear.

Here, a lifting operator was introduced such that Nitsche's methods' boundary integrals could be evaluated as volume integrals and rolled into the definition of element strain. An additional advantage occurs from this method in that some amount of stabilization naturally comes from the modified element strains. The method is shown to perform as well or better than a standard Nitsche's method in linear test problems with no additional stabilization. The discontinuous Galerkin version of Nitsche's method was applied to the case of embedded meshes for non-linear elasticity. Use of this method circumvented the need to find an expression for the stress variation - previously an outstanding question for non-linear applications on Nitsche's method. Optimal convergence in displacement and energy norms was

observed for all linear problems. Non-linear problems converged as well. In all cases, the discontinuous Galerkin method performed as well or better than either standard mortars or penalty methods.

\section{References}

[1] JD Sanders, TA Laursen, and MA Puso. A Nitsche embedded mesh method. Computational Mechanics. submitted for review.

[2] DN Arnold, F Brezzi, B Cockburn, and LD Marini. Unified analysis of discontinuous Galerkin methods for elliptical problems. SIAM Journal of Numerical Analysis, 39:17491779, 2002.

[3] FPT Baaijens. A fictitious domain/mortar element method for fluid-structure interaction. International Journal for Numerical Methods in Engineering, 35:743-761, 2001.

[4] AJ Lew and GC Buscaglia. A discontinuous-Galerkin based immersed boundary method. International Journal for Numerical Methods in Engineering, 76(4):427-454, 2008. 
[5] JL Steger and JA Benek. On the use of composite grid schemes in computational aerodynamics. Computer Methods in Applied Mechanics and Engineering, 64:301-320, 1987.

[6] A Hansbo, P Hansbo, and MG Larson. A finite element method on composite grids based on Nitsche's method. ESAIM: Mathematical Modeling and Numerical Analysis, $37: 209-225,2003$.

[7] J Nitsche. Über ein Variationsprinzip zur Lösung von Dirichlet-problemen bei Verwendung von Teilräumen, die keinen Randbedingungen unterworfen sind. Abhandlungen aus dem Mathematischen Seminar der Universität Hamburg, 36, 1971.

[8] Rolf Stenberg. On some techniques for approximating boundary conditions in the finite element method. Journal of Computational and Applied Mathematics, 63:139-148, 1995.

[9] JC Barbosa and TRJ Hughes. The finite element method with Lagrange multipliers on the boundary: circumventing the Babuška-Brezzi condition. Computer Methods in Applied Mechanics and Engineering, 85:109-128, 1991.

[10] M Griebel and MA Schweitzer. A particle-partition of unity method part v: boundary conditions. In Geometric Analysis and Nonlinear Partial Differential Equations, pages 519-546. Springer, 2002.

[11] S Fernández-Méndez and A Huerta. Imposing essential boundary conditions in mesh-free methods. Computer Methods in Applied Mechanics and Engineering, 193:1257-1275, 2004 .

[12] A Fritz, Hüeber, and BI Wohlmuth. A comparison of mortar and Nitsche techniques for linear elasticity. CALCOLO, 41:115-137, 2004.

[13] P Wriggers and G Zavarise. A formulation for frictionless contact problems using a weak form introduced by Nitsche. Computational Mechanics, 41:407-420, 2008.

[14] JE Dolbow and I Harari. An efficient finite element method for embedded interface problems. International Journal for Numerical Methods in Engineering, 78:229-252, 2009.

[15] A Hansbo and P Hansbo. An unfitted finite element method, based on Nitsche's method, for elliptical interface problems. Computer Methods in Applied Mechanics and Engineering, 191:5537-5552, 2002.

[16] WH Reed and TR Hill. Triangular mesh methods for the neutron transport equation. Technical Report LA-UR-73-479, Los Alamos Scientific Laboratory, 1973.

[17] F Brezzi, G Manzini, D Marini, P Pietra, and A Russo. Discontinuous Galerkin approximations for elliptical problems. Numerical Methods for Partial Differential Equations, 16:365-378, 2000. 
[18] F Bassi and S Rebay. A high-order accurate discontinuous finite element method for the numerical solution of the navier-stokes equations. Journal of Computational Physics, 131:267-279, 1997.

[19] GA Baker. Finite element methods for elliptical equations using nonconforming elements. Mathematics of Computation, 31:45-59, 1977.

[20] DN Arnold. An interior penalty finite element method with discontinuous elements. SIAM Journal of Numerical Analysis, 19:742-760, 1982.

[21] A Lew, P Neff, D Sulsky, and M Ortiz. Optimal bv estimates for a discontinuous Galerkin method in linear elasticity. Applied Mathematics Research Express, 3:73-106, 2004.

[22] R Gracie, H Wang, and T Belytschko. Blending in the extended finite element method by discontinuous Galerkin and assumed strain methods. International Journal for $\mathrm{Nu}$ merical Methods in Engineering, 74:1645-1669, 2008.

[23] A Noels and R Radovitzky. A general discontinuous Galerkin method for finite hyperelasticity. formulation and numerical applications. International Journal for Numerical Methods in Engineering, 68:64-97, 2006.

[24] A Ten Eyck and A Lew. Discontinuous Galerkin methods for non-linear elasticity. International Journal for Numerical Methods in Engineering, 67:1204-1243, 2006.

Prepared by LLNL under Contract DE-AC52-07NA27344. 